Artículo

\title{
Cambio climático y distribución potencial de frijol lima en Mesoamérica y Aridoamérica
}

\author{
Carlos Félix Barrera-Sánchez \\ José Ariel Ruiz-Corral ${ }^{\S}$ \\ Patricia Zarazúa-Villaseñor \\ Rogelio Lépiz-Ildefonso \\ Diego Raymundo González Eguiarte \\ Centro Universitario de Ciencias Biológicas y Agropecuarias-Universidad de Guadalajara. Camino Ing. \\ Ramón Padilla Sánchez núm. 2100. La Venta del Astillero, Zapopan, Jalisco, México. CP. 45110. \\ (carlosbarrera43@gmail.com; pzarazua@cucba.udg.mx; rlepiz@cucba.udg.mx; diegonz@cucba.udg.mx). \\ ${ }^{\S}$ Autor para correspondencia: ariel.ruiz@academicos.udg.mx.
}

\section{Resumen}

Los objetivos de esta investigación fueron caracterizar los descriptores ecológicos y evaluar los impactos del cambio climático en la distribución potencial del frijol lima Phaseolus lunatus L. en las regiones de Mesoamérica y Aridoamérica. Se integró una base de datos de sitios de ocurrencia tomando seis fuentes. A partir, de las coordenadas geográficas de dichos sitios de ocurrencia y mediante el uso de sistemas de información geográfica, se extrajeron valores de variables agroclimáticas derivados de rasters de los sistemas Worldclim, USGS/NASA SRTM y CGIAR. Así se integró una matriz de datos agroclimáticos mediante la cual se determinaron los descriptores ecológicos de Phaseolus lunatus. Se modeló la distribución de P. lunatus con el modelo Maxent. Los resultados mostraron que $P$. lunatus se adapta a una amplia gama de condiciones ambientales que incluye una condición hídrica que va de semiárida a húmeda, una condición térmica que oscila de templada a muy cálida, y tiene presencia en los rangos altitudinal, térmico y pluvial de 0-2 386 msnm, 13.2-29.9 ${ }^{\circ} \mathrm{C}$ y 400-4 $250 \mathrm{~mm}$, respectivamente. Estos descriptores señalan la existencia de poblaciones portadoras de genes resistentes a condiciones de estrés climático. El modelo Maxent modeló satisfactoriamente la distribución de Phaseolus lunatus en los diferentes escenarios climáticos evaluados (AUC= 0.94$)$. El cambio climático proyectado por el modelo HADGEM-ES para 2050 y 2070 y RCPs 4.5 y 8.5 es desfavorable para la distribución de $P$. lunatus, mientras que según el modelo CCMS4 el clima 2050 y 2070 es favorable en la ruta RCP4.5 y desfavorable en la ruta $\mathrm{RCP} 8.5$.

Palabras clave: adaptación, cambio climático, descriptores ecológicos, frijol lima.

Recibido: mayo de 2020

Aceptado: julio de 2020 


\section{Introducción}

Las modificaciones que el cambio climático está estableciendo sobre los patrones de temperatura, presión atmosférica, dirección del viento, humedad ambiental, disponibilidad de radiación solar, evapotranspiración y precipitación, conducen a una reconfiguración de los ambientes bajo los cuales las especies vegetales (cultivadas y silvestres) deben crecer y desarrollar (Teixeira et al., 2013; Durán et al., 2020). El cambio climático afecta de manera diferenciada a las distintas regiones del mundo, lo que incluye a las regiones de Aridoamérica y Mesoamérica.

Esto tiene implicaciones importantes, sobre todo porque Mesoamérica es considerada centro de origen y diversidad de plantas que hoy son importantes cultivos y fuente de alimentación para la civilización humana. Dentro de esta región, tan solo México es considerado como un centro primario de diversidad y domesticación de algunas especies cultivadas, como Phaseolus vulgaris (frijol común); P. acutifolius (frijol tépari); P. coccineus (frijol escarlata); $P$. dumosus (frijol anual) y P. lunatus (frijol lima) (Gepts y Debouck, 1991).

La caracterización de los descriptores ecológicos y el posible impacto del cambio climático sobre los cultivos y sus parientes silvestres, puede coadyuvar al diseño de estrategias de adaptación al cambio climático en los sistemas productivos agrícolas. Phaseolus lunatus L., es una especie propia de América, la cual presenta dos centros de domesticación: uno se ubica en los Andes alrededor de $2000 \mathrm{aC}$, donde se produjo una variedad de semilla grande (tipo Lima), mientras que el segundo se produce muy probablemente en Mesoamérica alrededor del año $800 \mathrm{aC}$, cuando se obtiene una variedad de semilla pequeña. Esta forma silvestre de semilla pequeña se encuentra distribuida desde México hasta Argentina, mientras que la variedad de semilla grande (tipo lima) se encuentra distribuida en el norte del Perú (Paredes et al., 2010).

Serrano-Serrano et al. (2012) evidenciaron la presencia de dos grupos genéticos silvestres de Phaseolus lunatus en Mesoamérica: uno (MI) que posee una distribución que se remite a la parte central del occidente de México, incluyendo la región costera del Pacífico y otro (MII) que tiene una distribución muy amplia y que ocurre hacia el Golfo de México, la Península de Yucatán, Centroamérica y Sudamérica. De acuerdo con estos autores y Martínez-Castillo et al. (2014) la región más probable de domesticación de P. lunatus del grupo MI es un área entre los estados de Jalisco, Michoacán y Guerrero, mientras que para el grupo MII es un área situada entre Guatemala y Costa Rica (Andueza-Noh et al., 2013; Martínez-Castillo et al., 2014).

La distribución de Phaseolus lunatus ha sido señalada con anterioridad en 17 tipos climáticos, con presencia tanto en la región de Mesoamérica como de Aridoamérica, evidenciando una dispersión amplia y gran variabilidad ambiental entre los hábitats de esta especie (López et al., 2005). Aunado a ello, las poblaciones silvestres de Phaseolus lunatus de la región Occidente de México muestran también amplia variabilidad morfológica (López-Alcocer et al., 2016).

Tanto la persistencia como la producción de biomasa de las poblaciones silvestres de Phaseolus lunatus, presentan alta dependencia de los niveles de lluvia año con año, los cuales están siendo modificados por el cambio climático (Durán et al., 2020), amenazando la permanencia de estos recursos fitogenéticos en su hábitat natural. Por lo anterior, la presente investigación tuvo como objetivos caracterizar los descriptores ecológicos y evaluar los impactos del cambio climático en 
la distribución potencial de Phaseolus lunatus en las regiones de Mesoamérica y Aridoamérica. La información generada podría coadyuvar con la planeación a futuro de la conservación y aprovechamiento de las poblaciones silvestres de $P$. lunatus.

\section{Materiales y métodos}

\section{Área de estudio}

El área de estudio comprende el sur de los Estados Unidos de América, México y Centroamérica (Figura 1), región que cubre $3946177.4 \mathrm{~km}^{2}$ y que culturalmente es conocida como las regiones de Mesoamérica y Aridoamérica (Kirchhoff, 1943).

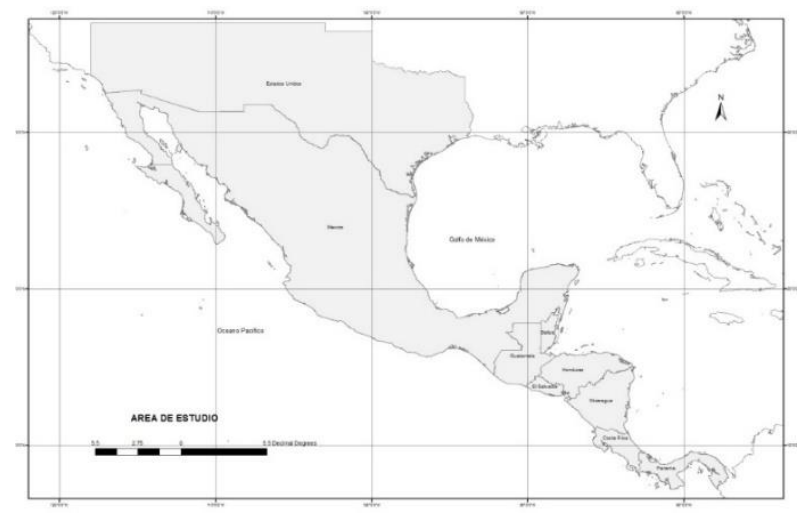

Figura 1. Área de estudio para Phaseolus lunatus.

\section{Datos de ocurrencia}

Se integró una matriz de datos de ocurrencia de Phaseolus lunatus a partir de los datos pasaporte georreferenciados con base en la información de las siguientes entidades: Centro Internacional de Agricultura Tropical (CIAT), Instituto Nacional de Ecología, Universidad de Guadalajara, Instituto Nacional de Investigaciones Forestales, Agrícolas y Pecuarias (INIFAP), Fraitag y Debouck (2002), así como datos particulares facilitados personalmente por el Dr. Rogelio Lépiz Ildefonso. Dr. Rogelio Lépiz Ildefonso. Profesor-Investigador, CUCBA, Universidad de Guadalajara.

\section{Variables climáticas}

Se utilizaron imágenes mensuales normales de temperatura máxima (12), temperatura mínima (12), temperatura media (12) y precipitación (12), además de imágenes de las 19 variables bioclimáticas, todas derivadas del sistema Worldclim y correspondientes al período 1960-2000 (Hijmans et al., 2005). Además, se utilizaron 12 imágenes mensuales normales de evapotranspiración potencial anual (ETP) que se obtuvieron de la base de datos global geoespacial de balance hídrico de suelo del CGIAR (Trabuco y Zomer, 2010). También se agregaron como variables de análisis la longitud, la latitud y la altitud de los sitios de presencia. Para ello se tomó la imagen de altitud del sitio USGS/NASA SRTM (Jarvis, 2008). Con los datos de precipitación y ETP mensual se generaron 12 imágenes mensuales de índice de humedad (IH) mediante la expresión IH= P/ETP. Toda esta información se trabajó con una resolución dada por un tamaño de celda de 30 segundos de arco. En total se analizaron 94 variables. 


\section{Caracterización climática de sitios de presencia de Phaseolus lunatus}

Se utilizó la información de coordenadas geográficas de los sitios de ocurrencia de frijol lima y las imágenes climáticas y bioclimáticas del sistema Worldclim para caracterizar la climatología de los sitios de distribución de esta especie. Para ello se utilizó el procedimiento de extracción de información del programa ArcGis (ESRI, 2010). La información extraída se incorporó a la matriz de datos de ocurrencia de P. lunatus en la hoja de cálculo Excel de Microsoft. Finalmente se caracterizó el tipo climático de los sitios de presencia mediante el sistema que proponen Ruiz et al. (2018) y que refieren de manera ilustrativa Sánchez et al. (2018), en el que son teóricamente posibles 36 ambientes, producto de la combinación de seis zonas hídricas, determinados por intervalos de un índice de humedad ( $\mathrm{IH}=$ precipitación anual/evapotranspiración potencial anual) e intervalos de temperatura media anual (Tma), respectivamente (Cuadro 1).

Cuadro 1. Zonas hídricas y zonas térmicas para caracterizar tipos de ambientes agroclimáticos.

\begin{tabular}{cccc}
\hline Intervalos de IH & Zona hídrica & Intervalos de Tma $\left({ }^{\circ} \mathrm{C}\right)$ & Zona térmica \\
\hline$<0.05$ & Hiperárida & $<5$ & Fría \\
$0.05-0.2$ & Árida & $5-12$ & Semifría \\
$0.20-0.5$ & Semiárida & $12-18$ & Templada \\
$0.5-0.65$ & Subhúmeda seca & $18-22$ & Semicálida \\
$0.65-1$ & Subhúmeda húmeda & $22-26$ & Cálida \\
1 & Húmeda & $=>26$ & Muy cálida \\
\hline
\end{tabular}

\section{Análisis estadístico}

A partir de la matriz de sitios de ocurrencia caracterizada climáticamente, se realizó un análisis de correlación múltiple para depurar la lista de variables climáticas. Se utilizó el criterio de coeficiente de correlación $\geq 0.95$ para la eliminación de variables. Con la lista depurada de variables se procedió a realizar un análisis de componentes principales e identificar las variables de mayor peso estadístico en la presencia de frijol lima. Para este análisis se empleó el programa NTsys 3.3.0 (Rohlf, 2009).

\section{Descriptores climáticos}

A partir, de las variables climáticas seleccionadas para el análisis y considerando la caracterización climática de la matriz de datos de ocurrencia, se dedujeron los descriptores ecológicos de frijol lima, para lo cual se consideraron los valores mínimos, máximos y promedio de cada variable.

\section{Modelación de nichos potenciales de distribución de Phaseolus lunatus}

Previo a la modelación de nichos potenciales de distribución, se aplicó una prueba de aleatoriedad para determinar si los sitios de ocurrencia de Phaseolus lunatus están distribuidos al azar (Bivand et al., 2014). Esta prueba se corrió utilizando un programa implementado en R. Se utilizó el modelo Maxent (Phillips et al., 2006) para modelar la distribución potencial de frijol lima bajo el escenario climático actual o de referencia y bajo los escenarios climáticos año 2050 y año 2070. 
Para la modelación de la distribución potencial de $P$. lunatus en escenarios futuros o de cambio climático se emplearon los datos derivados de los modelos de circulación general: HadGEM2ES (European Network for Earth System-MetOffice Hadley Centre) y CCSM4 Community Earth System Model (CCSM4) bajo dos rutas de concentración de gases efecto invernadero (RCP) 4.5 y 8.5. El primer modelo, desarrollado en el Reino Unido, incorpora elementos que permiten modelar adecuadamente la vegetación continental pues incluye diversos tipos de vegetación: árboles de hoja ancha, arboles de acículas, hierbas $\mathrm{C}_{3}$ y $\mathrm{C}_{4}$, así como arbustos (Bellouin et al., 2007).

El segundo modelo está desarrollado en Estados Unidos de América por el National Center for Atmospheric Research (NACAR) el cual incorpora la cobertura vegetal como uno de sus cuatro componentes acoplados. Estos modelos han sido evaluados estadísticamente por Conde et al. (2011), concluyen que el Modelo CCSM es uno de los que mejor describe el clima de la región de México y Centroamérica, en tanto que HadGEM ya ha sido utilizado para estudios de la vegetación de México con buenos resultados (INE, 2009).

El modelo Maxent ha sido descrito como especialmente eficiente en el manejo de interacciones complejas entre variables de respuesta y variables predictoras por ser un modelo robusto aún en muestras pequeñas de datos (Fourcade et al., 2014). Maxent utiliza el principio de máxima entropía sobre datos de presencia de la especie para estimar un grupo de funciones que relacionen variables ambientales con idoneidad de hábitat para aproximar la distribución geográfica potencial de la especie (Phillips et al., 2006). Maxent representa la distribución de una especie; a través, de una función de probabilidad $\mathrm{P}$ sobre un conjunto $\mathrm{X}$ de sitios en el área de estudio. La función de probabilidad para $i$ variables ambientales se expresa de la siguiente manera.

$P(x)=e \lambda \cdot f(x) / Z \lambda$. Donde: $\mathrm{P}(\mathrm{x})=$ función de probabilidad; $\lambda=$ vector de coeficientes de ponderación; $\mathrm{f}=$ vector correspondiente de funciones de variables ambientales; $\mathrm{Z}=$ constante de normalización para asegurar que $\mathrm{P}(\mathrm{x})=1$. Los valores $\mathrm{P}(\mathrm{x})$ así obtenidos, representan valores de idoneidad relativa para la presencia de la especie, constituyendo así la base de un modelo de distribución potencial.

En la modelación de la distribución potencial de Phaseolus lunatus con Maxent se utilizaron los parámetros por defecto, mediante la réplica de ejecución crossvalidate con 1000 repeticiones. Se destinó 50\% de los datos para entrenamiento y 50\% para prueba. En escenarios de cambio climático, esta configuración se mantuvo. Finalmente se generó un mapa binario presencia/ausencia de Phaseolus lunatus utilizando como método de umbral de corte, aquél que presentara una tasa de omisión cercana a $0 \%$ pero con el valor de corte más alto (Sánchez et al., 2018).

\section{Resultados y discusión}

\section{Adaptación climática de Phaseolus lunatus}

En la Figura 2, se puede observar la distribución de Phaseolus lunatus en las distintas zonas agroclimáticas del área de estudio. Como puede observarse en el mapa de la Figura 2 y Cuadro 2, esta especie está presente en 17 de las 26 zonas agroclimáticas del área de estudio. Sin embargo, de las 17 zonas agroclimáticas en las que ocurre $P$. lunatus, dos corresponden a zonas áridas. 
Las zonas áridas difícilmente pueden albergar el ciclo de desarrollo de una leguminosa como $P$. lunatus, ya que los niveles de precipitación anual en esos ambientes, es mucho menor que $500 \mathrm{~mm}$, valor señalado como el umbral pluvial mínimo de adaptación de esta especie (Baudoin, 1989). La presencia de $P$. lunatus en zonas áridas en el área de estudio muy probablemente se debe a que tiene acceso al recurso hídrico, pero no a través de precipitaciones pluviales.

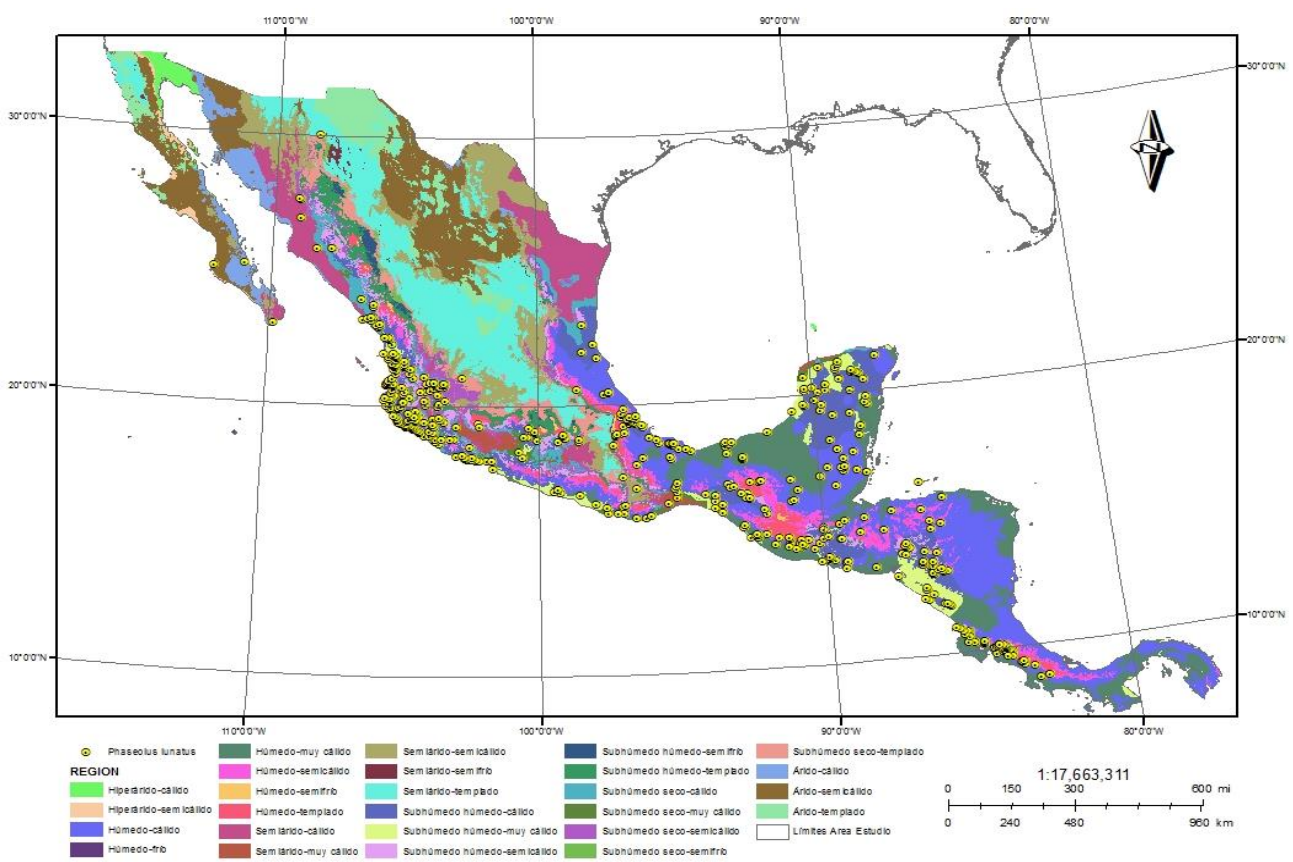

Figura 2. Regiones agroclimáticas y distribución de Phaseolus lunatus.

Cuadro 2. Distribución de Phaseolus lunatus por región agroclimática.

\begin{tabular}{lcccc}
\hline Región Agroclimática & Registros & $(\%)$ & $(\%)$ por índice de humedad & $(\%)$ por índice térmico \\
\hline Subhúmedo húmedo-muy cálido & 63 & 10.7 & Subhúmedo húmedo & Muy cálidos \\
Subhúmedo húmedo-cálido & 150 & 25.5 & & \\
Subhúmedo húmedo-semicálido & 37 & 6.3 & & \\
Subhúmedo húmedo-templado & 2 & 0.3 & 42.9 & 26.2 \\
Húmedo-muy cálido & 58 & 9.9 & Húmedos & Cálidos \\
Húmedo-cálido & 92 & 15.6 & & \\
Húmedo-semicálido & 78 & 13.3 & & 47.1 \\
Húmedo-templado & 13 & 2.2 & 41 & Semicálido \\
Subhúmedo seco-muy cálido & 29 & 4.9 & Subhúmedo seco & \\
Subhúmedo seco-cálido & 27 & 4.6 & & Templado \\
Subhúmedo seco-semicálido & 20 & 3.4 & 12.9 & \\
Semiárido-muy cálido & 4 & 0.7 & Semiárido & \\
Semiárido-cálido & 7 & 1.2 & & 2.7 \\
Semiárido-semicálido & 5 & 0.9 & & 100 \\
Semiárido-templado & 1 & 0.2 & 2.9 & \\
Árido-cálido & 1 & 0.2 & Árido & \\
Árido-semicálido & 1 & 0.2 & 0.3 & \\
Total general & 588 & 100 & 100 & \\
\hline
\end{tabular}


En el Cuadro 2 se observa que el mayor número de sitios de ocurrencia de Phaseolus lunatus se produce en la zona agroclimática subhúmeda-húmeda cálida, señalando que este es el ambiente climático que se aproxima más a las condiciones climáticas óptimas para esta planta.

Sin considerar la zona hídrica árida, $P$. lunatus es capaz de distribuirse en cuatro zonas hídricas (desde semiárida hasta húmeda) y cuatro zonas térmicas (desde templada a muy cálida) aunque parece preferir el intervalo de subhúmeda-húmeda a húmeda y el intervalo de semicálida a muy cálida. La distribución de esta leguminosa en condiciones semiárida y templada es definitivamente muy reducida (Cuadro 2).

\section{Descriptores ecológicos}

El Cuadro 3 muestra los descriptores ecológicos de Phaseolus lunatus a partir, de las variables climáticas más representativas. Como puede verse, aun cuando esta leguminosa tiene preferencia por ambientes semicálidos, cálidos y muy cálidos (Cuadro 2), su presencia en altitudes de 0 a 2 $386 \mathrm{~m}$ (Cuadro 3) señala una amplia capacidad de adaptación, pues térmicamente puede distribuirse desde zonas templadas (valor mínimo de $13.2{ }^{\circ} \mathrm{C}$ de temperatura media anual, Cuadro 3) incluso en ambientes templados (Cuadro 2). El rango altitudinal encontrado coincide de manera aproximada con el reportado por López et al. (2005) de 0 a $2059 \mathrm{~m}$ y difiere un poco del señalado por Baudoin (1989) de 800 a 2000 msnm, así como del reportado por Debouck (2020) de 50-2 800 m. Estas diferencias seguramente se deben a las diferentes áreas geográficas consideradas por estos autores.

Cuadro 3. Descriptores ecológicos de Phaseolus lunatus.

\begin{tabular}{lccc}
\hline Variable & Mínimo & Máximo & Promedio \\
\hline Altitud & 0 & 2386 & 605 \\
Estación de crecimiento EC (días) & 83 & 365 & 212 \\
Temperatura media EC $\left({ }^{\circ} \mathrm{C}\right)$ & 13.2 & 29.9 & 24.4 \\
Temperatura máxima media EC $\left({ }^{\circ} \mathrm{C}\right)$ & 17.3 & 37 & 29.7 \\
Temperatura mínima media EC $\left({ }^{\circ} \mathrm{C}\right)$ & 8 & 24.2 & 19.1 \\
Precipitación acumulada promedio EC $(\mathrm{mm})$ & 250 & 4250 & 1368 \\
Índice de humedad promedio EC & 0.51 & 3.09 & 1.42 \\
Temperatura media anual $\left({ }^{\circ} \mathrm{C}\right)$ & 13.2 & 29.2 & 23.5 \\
Temperatura máxima media anual $\left({ }^{\circ} \mathrm{C}\right)$ & 17.3 & 36.1 & 29.6 \\
Temperatura mínima media anual $\left({ }^{\circ} \mathrm{C}\right)$ & 4.5 & 22.6 & 17.5 \\
Temperatura máxima del mes más cálido $\left({ }^{\circ} \mathrm{C}\right)$ & 18.4 & 40.1 & 32 \\
Temperatura mínima del mes más frío $\left({ }^{\circ} \mathrm{C}\right)$ & -3.7 & 21 & 14.4 \\
Precipitación anual $(\mathrm{mm})$ & 400 & 4250 & 1466 \\
Índice de humedad anual & 0.23 & 2.49 & 0.89 \\
\hline
\end{tabular}

De acuerdo con el Cuadro 3, el intervalo de estación de crecimiento en el que ocurre Phaseolus lunatus (83 a 365 días) coincide con el ciclo de desarrollo que Debouck (2020) reporta de 90 a 365 días para $P$. lunatus. 
Este intervalo de 83 a 365 días de estación de crecimiento, permite deducir que esta planta se adapta a ambientes con temporada de lluvias corta tanto como a ambientes de lluvia prácticamente todo el año, con una precipitación acumulada en el período de crecimiento de 250 a $4250 \mathrm{~mm}$, por lo que algunas poblaciones de $P$. lunatus presentes en estas condiciones extremas de humedad podrían estar indicando la existencia de poblaciones portadoras de genes resistentes a estas condiciones, como lo sugiere Maxted et al. (2013).

De igual manera, para el caso de la temperatura, $P$. lunatus se adapta a estaciones de crecimiento con una temperatura mínima promedio de $8{ }^{\circ} \mathrm{C}$ tanto como a estaciones de crecimiento donde la temperatura máxima puede promediar incluso $37{ }^{\circ} \mathrm{C}$. Las poblaciones representativas de estos valores térmicos también pueden resultar de interés por su posible genética de adaptación a temperaturas extremas. Más aún, de acuerdo con el Cuadro 3, existen poblaciones de Phaseolus lunatus que experimentan el mes más cálido con temperaturas máximas por arriba de $40{ }^{\circ} \mathrm{C}$ y otras que presentan el mes más frío con temperaturas por debajo de $0{ }^{\circ} \mathrm{C}$, sobre todo las formas de la especie de ciclo biológico largo, de hábito de crecimiento indeterminado (Debouck, 2020).

Es interesante destacar que los reportes sobre $P$. lunatus señalan un rango térmico de 16 a $26{ }^{\circ} \mathrm{C}$ (Debouck, 2020), en el presente estudio el intervalo obtenido es más amplio, 13.2 a $29.2{ }^{\circ} \mathrm{C}$ en escala anual y 13.2 a $29.9{ }^{\circ} \mathrm{C}$ a nivel de la estación de crecimiento (Cuadro 3). Estos valores constituyen límites térmicos de adaptación no reportados hasta hoy para $P$. lunatus, lo cual podría indicar que esta especie ha tenido la capacidad de ir explorando áreas más extremosas térmicamente al paso del tiempo.

De igual forma, la presente investigación obtuvo un límite superior de precipitación acumulada anual de $4250 \mathrm{~mm}$, cantidad que supera reportes previos como el de Debouck (2020) de 2800 mm. Finalmente, de acuerdo con el Cuadro 3, el índice de humedad promedio durante el ciclo de producción señala como límites inferior y superior de adaptación de P. lunatus, 0.51 y 3.09, respectivamente. De estos descriptores no existe referencia antecedente, debido a que variables como evapotranspiración, balance de humedad e índice de humedad no son típicamente parámetros considerados en la descripción de la ecología de cultivos.

\section{Modelación de la distribución potencial de Phaseolus lunatus}

La prueba de aleatoriedad reportó que los puntos de ocurrencia de Phaseolus lunatus tienen una distribución aglomerada y no una distribución aleatoria, por lo cual se estimaron las distancias entre los puntos de ocurrencia y se re-muestrearon los datos mediante el programa ArcGis (ESRI, 2010), por medio de una malla construida a partir de las distancias geográficas para obtener muestras aleatoriamente distribuidas. Con estas muestras se realizó la modelación de áreas potenciales de distribución de P. lunatus.

Del total de 94 variables que se consideraron en el presente estudio, el análisis de correlación múltiple y el análisis de componentes principales permitieron reducir la lista a 29 variables con la mayor significancia en la distribución de Phaseolus lunatus. En el Cuadro 4 se muestra el valor de los Eigenvectores de las 29 variables climáticas con mayor significancia en la distribución de $P$. lunatus en los tres primeros componentes principales. 
Cuadro 4. Eigenvectores de 29 variables climáticas a partir del análisis de componentes principales de sitios de ocurrencia de Phaseolus lunatus.

\begin{tabular}{|c|c|c|c|}
\hline Variable climática & CP1 & $\mathrm{CP} 2$ & CP3 \\
\hline Precipitación mensual de noviembre (mm) & -0.9335 & 0.0903 & 0.0962 \\
\hline Precipitación de noviembre a abril $\left({ }^{\circ} \mathrm{C}\right)$ & -0.9107 & 0.137 & 0.3526 \\
\hline Precipitación anual (mm) & -0.9007 & 0.084 & -0.2181 \\
\hline Precipitación mensual de octubre (mm) & -0.8996 & 0.1323 & -0.2914 \\
\hline Precipitación mensual de abril (mm) & -0.86 & -0.0146 & -0.1238 \\
\hline Precipitación mensual de mayo (mm) & -0.8179 & -0.0359 & -0.4224 \\
\hline Precipitación mensual de marzo (mm) & -0.8047 & 0.1769 & 0.3933 \\
\hline Precipitación del trimestre más seco (mm) & -0.7794 & 0.1437 & 0.5514 \\
\hline Precipitación del trimestre más frío (mm) & -0.7541 & 0.1796 & -0.0174 \\
\hline Precipitación mensual de junio (mm) & -0.7339 & -0.0043 & -0.4235 \\
\hline Precipitación mensual de diciembre (mm) & -0.7205 & 0.1645 & 0.5612 \\
\hline Precipitación mensual de febrero (mm) & -0.7014 & 0.1974 & 0.6448 \\
\hline Precipitación mensual de septiembre (mm) & -0.6491 & 0.1646 & -0.4407 \\
\hline Precipitación del trimestre más lluvioso (mm) & -0.6179 & 0.071 & -0.3424 \\
\hline Rango de temperatura media diurna $\left({ }^{\circ} \mathrm{C}\right)$ & 0.6525 & -0.2517 & -0.0006 \\
\hline Rango de temperatura anual $\left({ }^{\circ} \mathrm{C}\right)$ & 0.6999 & -0.2362 & 0.3352 \\
\hline Estacionalidad de la precipitación & 0.7619 & -0.1008 & -0.3279 \\
\hline Temperatura promedio del trimestre más frío $\left({ }^{\circ} \mathrm{C}\right)$ & 0.1622 & 0.946 & -0.244 \\
\hline Temperatura mínima de noviembre $\left({ }^{\circ} \mathrm{C}\right)$ & 0.063 & 0.9392 & -0.11 \\
\hline Temperatura mínima de abril $\left({ }^{\circ} \mathrm{C}\right)$ & -0.1014 & 0.9384 & -0.0065 \\
\hline Temperatura media anual $\left({ }^{\circ} \mathrm{C}\right)$ & 0.3378 & 0.9356 & 0.0026 \\
\hline Temperatura media mensual de abril $\left({ }^{\circ} \mathrm{C}\right)$ & 0.2216 & 0.9308 & 0.0314 \\
\hline Temperatura mínima del mes más frío $\left({ }^{\circ} \mathrm{C}\right)$ & -0.1693 & 0.9303 & -0.2007 \\
\hline Temperatura máxima de agosto $\left({ }^{\circ} \mathrm{C}\right)$ & 0.5057 & 0.8002 & 0.1607 \\
\hline Temperatura máxima de noviembre a abril $\left({ }^{\circ} \mathrm{C}\right)$ & 0.4883 & 0.7791 & -0.1814 \\
\hline Temperatura máxima del mes más cálido $\left({ }^{\circ} \mathrm{C}\right)$ & 0.5855 & 0.694 & 0.1583 \\
\hline Precipitación mensual de enero (mm) & -0.5822 & 0.2024 & 0.6881 \\
\hline Estacionalidad de la temperatura & 0.515 & -0.0099 & 0.6734 \\
\hline Isotermalidad & -0.3625 & -0.0326 & -0.7448 \\
\hline
\end{tabular}

Se observa entonces que en el primer componente se agrupan variables que tienen que ver con el factor humedad (precipitación), en el segundo componente, variables que tienen que ver con el factor energético (temperatura) y en el tercer componente, otro tipo de variables que se derivan de la interacción de ambos factores. 
Al correr el análisis Maxent con las 29 variables se obtuvo un modelo cuya curva operacional o curva ROC reportó un área bajo la curva (AUC) de 0.94, que de acuerdo con Araújo et al. (2005) representa un ajuste excelente del modelo y por tanto permite una buena discriminación de áreas adecuadas contra áreas no adecuadas para Phaseolus lunatus (Thuiller et al., 2009) al elaborar un mapa binomial.

El análisis Jackknife permitió identificar las variables de mayor contribución a la modelación de la distribución de Phaseolus lunatus. El Cuadro 5 muestra la contribución de las 11 variables que más aportan a este respecto. Tanto Bio04, como Prec09 y Bio16 se mantuvieron como las tres variables de mayor contribución a la distribución de esta leguminosa en todas las climatologías analizadas, tanto en la de referencia, como en las climatologías de cambio climático.

Cuadro 5. Porcentaje de contribución de 11 variables climáticas que más aportan para la modelación de la distribución de Phaseolus lunatus.

\begin{tabular}{lcc}
\hline \multicolumn{1}{c}{ Variable } & $\begin{array}{c}\text { Porcentaje de } \\
\text { contribución }\end{array}$ & $\begin{array}{c}\text { Importancia de la } \\
\text { permutación }\end{array}$ \\
\hline Estacionalidad de la temperatura (Bio04) & 48.3 & 51.9 \\
Precipitación del mes de septiembre (Prec09) & 13.3 & 3.6 \\
Precipitación del trimestre más húmedo (Bio16) & 6 & 3.8 \\
Estacionalidad de la precipitación (Bio15) & 4.3 & 2 \\
Precipitación del mes de mayo (Prec05 & 3.8 & 3.7 \\
Precipitación del mes de diciembre (Prec12) & 3.3 & 2.9 \\
Rango de temperatura anual (Bio07) & 2.1 & 2.9 \\
Temperatura promedio del trimestre más frío (Bio11) & 2.1 & 0.1 \\
Precipitación del mes de febrero (Prec02) & 2 & 0 \\
Temperatura mínima del mes más frío (Bio06) & 2 & 0.9 \\
Temperatura mínima del mes de noviembre (Tmin11) & 1.7 & 8 \\
\hline
\end{tabular}

El análisis de la evolución que tendrían estas tres variables en las climatologías de cambio climático se presenta en la Figura 3. Ahí se puede ver que Bio04 tiende a incrementar en los escenarios de cambio climático. Prec09 en cambio tiende a disminuir, con un porcentaje de $-1 \%$ en la climatología 2050 RCP4.5 modelo CCMS4, a 15\% en la climatología 2070 RCP8.5 modelo HADGEM-ES.

Con respecto a Bio16 el modelo CCMS4 estima una reducción tanto al pasar del año 2050 a 2070 como al cambiar del RCP 4.5 a 8.5, mientras que el modelo HADGEM-ES estima un valor estable de Bio16. 


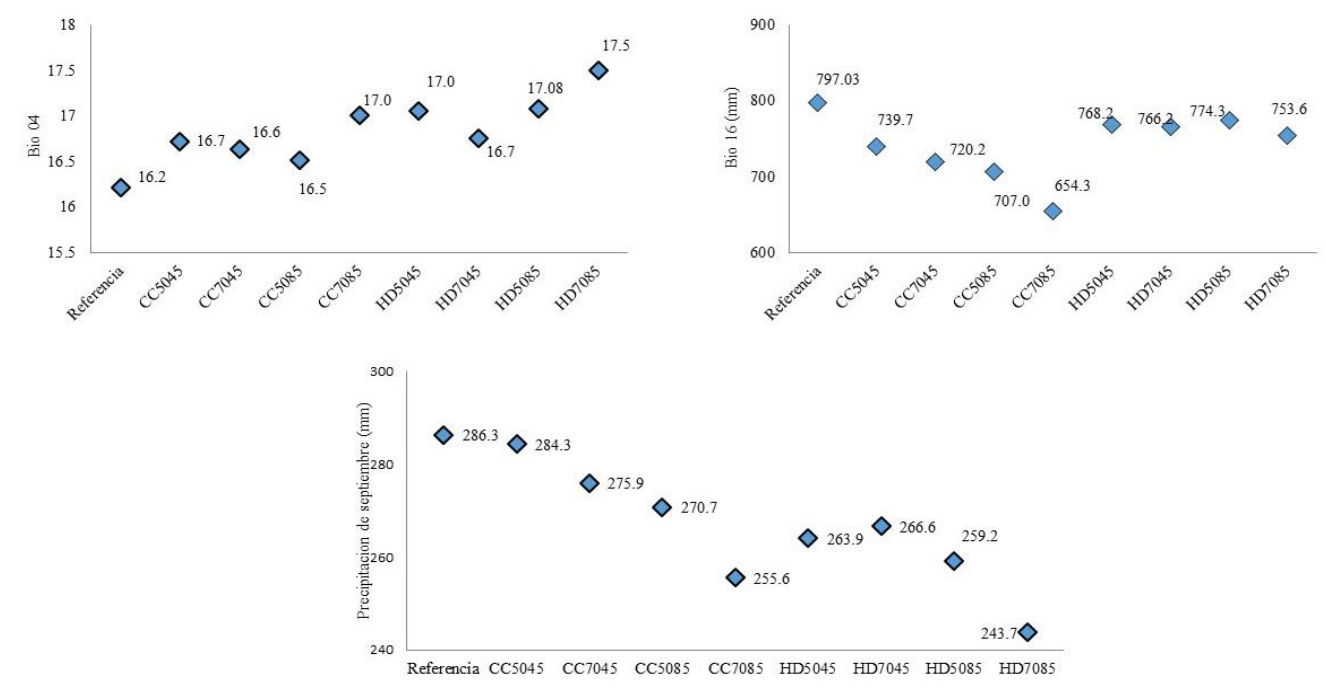

Figura 3. Evolución esperada de tres variables en diversos escenarios de cambio climático.

El mapa binario para el escenario de referencia reveló que, a gran escala, la distribución actual de Phaseolus lunatus coincide con la distribución potencial simulada por el modelo Maxent (Figura 4). Sin embargo, a menor escala son visibles áreas de distribución potencial que carecen de sitios de ocurrencia actual de esta leguminosa. Esto indica la presencia de sitios adecuados para la futura exploración y posible colecta de nuevas poblaciones de $P$. lunatus.

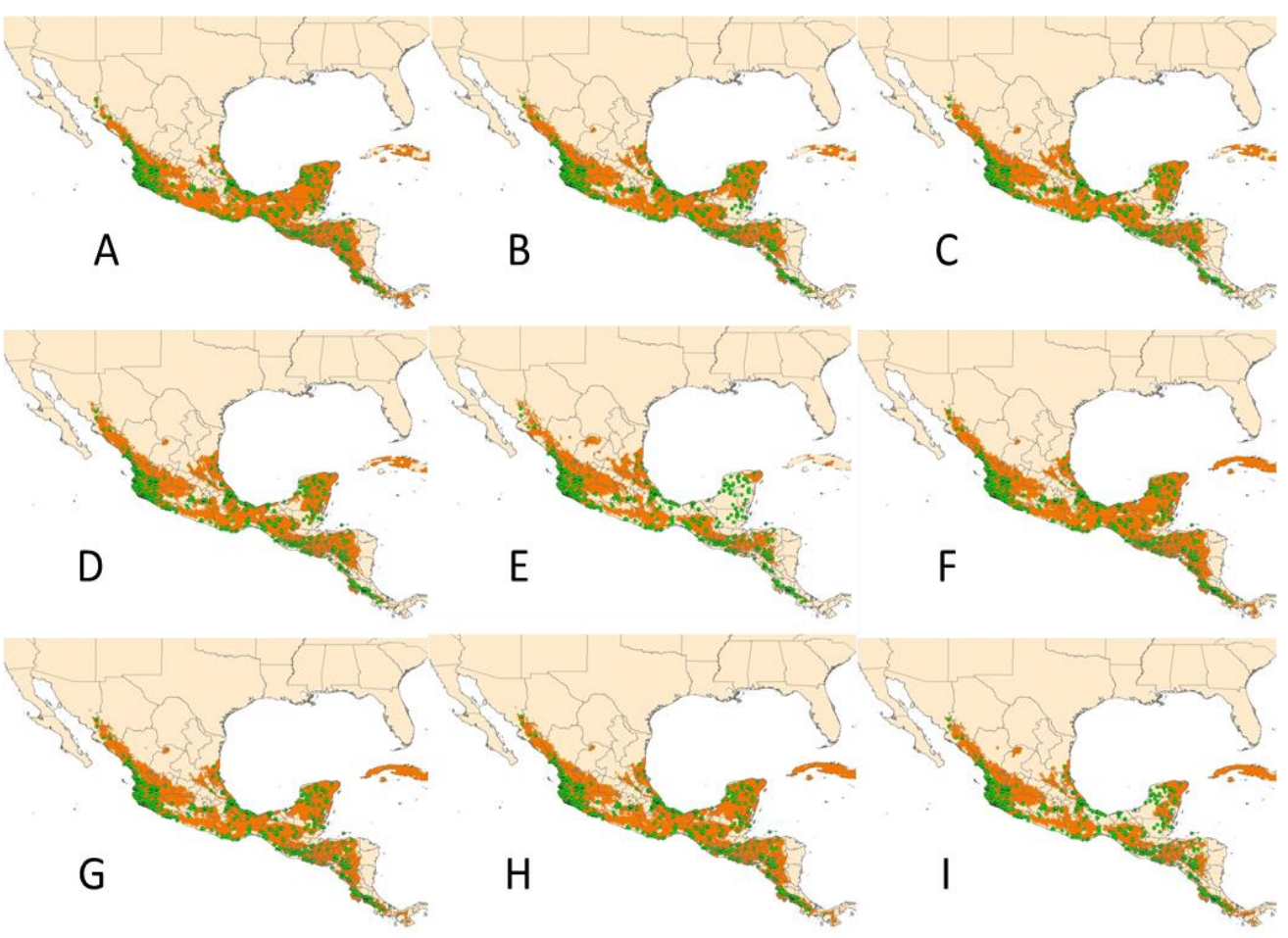

Figura 4. Distribución actual (verde) y potencial (naranja) de Phaseolus lunatus en diversas climatologías: A) 1961-2010; B) HADGEM-ES RCP4.5 2050; C) HADGEM-ES RCP4.5 2070; D) HADGEM-ES RCP8.5 2050; E) HADGEM-ES RCP8.5 2070; F) CCMS4 RCP4.5 2050; G) CCMS4 RCP4.5 2070; H) CCMS4 RCP8.5 2050; I) CCMS4 RCP8.5 2070. 
En las climatologías de cambio climático, el modelo HADGEM-ES en los dos RCPs señala un descenso en la superficie de distribución potencial de $P$. lunatus principalmente en la Península de Yucatán y Centroamérica (Figuras 4B, 4C, 4D y 4E) que va desde -8 a $-10 \%$ en el RCP4.5 a un intervalo de -13 a $-37 \%$ (Figura 5) en el RCP8.5. Estos efectos sobre la Península de Yucatán son particularmente importantes, debido a que esta zona representa uno de los centros de diversidad más importantes del grupo genético MII de frijol lima.

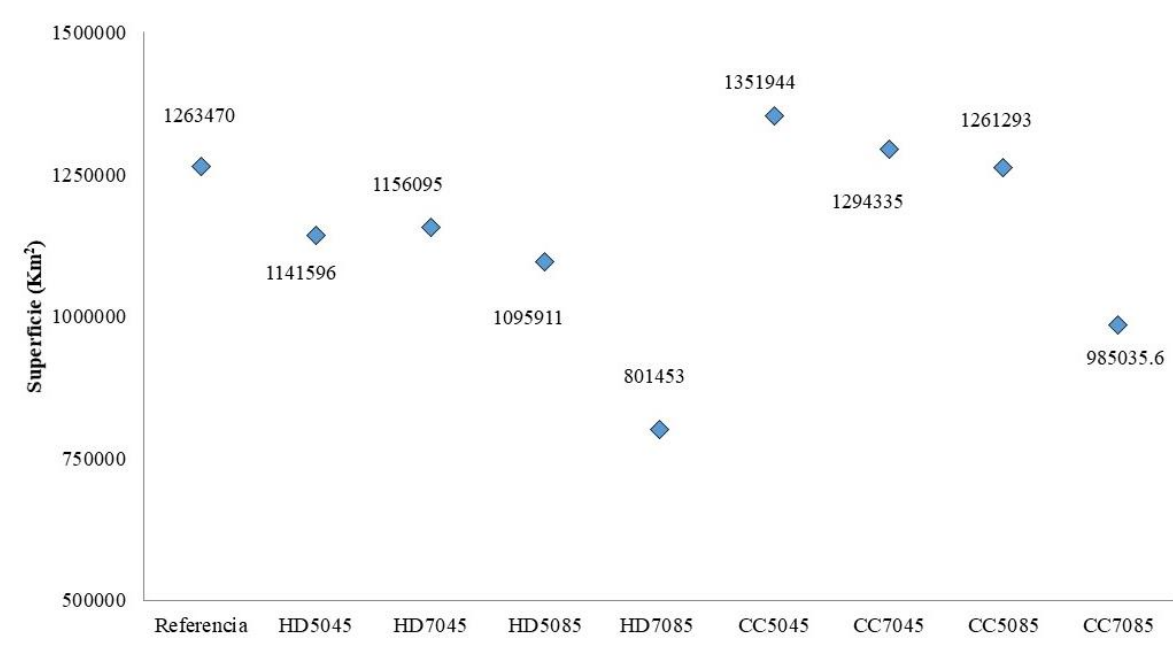

Figura 5. Efecto del cambio climático en la superficie potencial de distribución de $P$. lunatus.

Desde el escenario 2050 RCP4.5 (Figura 4B) se advierte una disminución de áreas potenciales para esta planta, en dirección de sur a norte de la Península de Yucatán, esto es norte de Guatemala y Campeche, lo cual se va acentuando en los siguientes escenarios (Figuras 4C, 4D y 4E) abarcando cada vez mayor superficie de este territorio, hasta reducir las áreas potenciales a una pequeña zona en el extremo noreste de la Península, en el estado de Yucatán (Figura 4E).

En el caso del modelo CCMS4, las Figuras 4F, 4G, 4H y 4I muestran la secuencia de mapas de áreas potenciales de distribución del frijol lima. En ella se puede ver que el RCP4.5 representa condiciones ambientales favorables, que causarían un incremento de $7 \%$ en el año 2050 y $+2 \%$ en 2070 (Figura 5), ocurriendo principalmente en la región norte-centro y occidente de México, además de El Caribe (Figuras 4F y 4G), lo cual podría en un futuro dar lugar a la presencia de nuevas poblaciones de frijol lima en estas regiones y con ello una nueva configuración de la distribución de esta especie en el área de estudio. Mientras que el RCP8.5 propiciaría una reducción en la superficie potencial de frijol lima en la Península de Yucatán y Centroamérica (Figura 4) de $-0.17 \%$ en 2050 a $-22 \%$ en 2070 (Figura 5). Estos resultados positivos y negativos para P. lunatus concuerdan con lo reportado para Tithonia diversifolia en México por Durán et al. (2020).

\section{Conclusiones}

El mayor número de sitios de ocurrencia de Phaseolus lunatus se produce en la zona agroclimática subhúmeda-húmeda cálida, señalando una condición climática óptima para esta especie. Sin embargo, $P$. lunatus tiene presencia en cuatro zonas hídricas: semiárida, subhúmeda-seca, subhúmeda-húmeda y húmeda y cuatro zonas térmicas: templada, semicálida, cálida y muy cálida. 
Los descriptores ecológicos de Phaseolus lunatus, señalan que esta planta se adapta a una gama amplia de condiciones ambientales, como lo evidencian un rango altitudinal de 0-2 $386 \mathrm{msnm}$, un rango térmico anual de $13.2-29.9^{\circ} \mathrm{C}$ y un rango pluvial anual de $400-4250 \mathrm{~mm}$.

Estos descriptores permiten deducir la existencia de poblaciones portadoras de genes resistentes a condiciones climáticas extremas. El modelo Maxent permitió modelar satisfactoriamente la distribución de Phaseolus lunatus. Las variables que más contribuyen a explicar la distribución de esta especie son Bio04 (estacionalidad de la temperatura), precipitación del mes de septiembre y Bio16 (precipitación del trimestre más húmedo). El cambio climático proyectado por el modelo HADGEM-ES para 2050 y 2070 y RCPs 4.5 y 8.5 es desfavorable para la distribución de $P$. lunatus, mientras que según el modelo CCMS4 el clima 2050 y 2070 es favorable en la ruta RCP4.5 y desfavorable en la ruta RCP8.5.

\section{Literatura citada}

Andueza-Noh, R. H.; Serrano-Serrano, M. L.; Chacón-Sánchez, M. I.; Sánchez del Pino, I.; Camacho-Pérez, L.; Coello-Coello, J.; Mijangos C. J.; Debouck, D. G. and MartínezCastillo, J. 2013. Multiple domestications of the Mesoamerican gene pool of lima bean (Phaseolus lunatus L.): evidence from chloroplast DNA sequences. Gen. Res. Crop Evol. 60(3):1069-1086.

Araújo, M.; Pearson, R.; Thuiller, W. and Erhard, M. 2005. Validation of species-climate impact models under climate change. Global Change Biol. 11(9):1504-1515.

Baudoin, J. P. 1989. Phaseolus lunatus L. In: plant resources of South-East Asia No. 1: Pulses (Ed.). By Maesen, L. J. G. van der \Somaatmadja]. Wageningen, The Netherlands: Pudoc. 57-60. pp.

Bellouin, N.; Boucher, O.; Haywood, J.; Johnson, C.; Jones, A.; Rae, J. and Woodward, S. 2007. Improved representation of aerosols for HadGEM2. Hadley Centre Technical Note 73, Met Office Hadley Centre. Exeter, EXI 3PB. United Kigdom. 42 p.

Bivand, R. S.; Pebesma, E. J. and Gómez-Rubio, V. 2014. Applied spatial data analysis with R. Doi: 10.1007/978-1-4614-7618-4. Springer. Switzerland. 405 p.

Conde, C.; Estrada, F.; Martinez, B.; Sánchez, O. and Gay, C. 2011. Regional climate change scenarios for México. Atmósfera. 24(1):125-140.

Debouck, D. G. 2020. La agricultura en Mesoamérica. Organización de las Naciones Unidas para la Agricultura y la Alimentación (FAO). http://www.fao.org/tempref/GI/Reserved/ FTP _ FaoRlc/old/prior/segalim/prodalim/prodveg/cdrom/contenido/libro09/Cap2_2.htm\#auto.

Durán, P. N.; Loya, O. J. L.; Ruiz, C. J. A.; González, E. D. R.; García, P. J. D.; Martínez, G. S. y Crespo, G. M. R. 2020. Impacto del cambio climático en la distribución potencial de Tithonia diversifolia (Hemsl.) A. Gray en México. Rev. Mex. Cienc. Pecu. 11(supl 2):93106. https://doi.org/10.22319/rmcp.v11s2.4705.

ESRI. 2010. ArcGis Version 10.0. Environmental Systems Research Institute, Inc., Redlands, CA.

Fourcade, Y.; Engler, J. O.; RoĖdder, D. and Secondi, J. 2014. Mapping species distributions with Maxent using a geographically biased sample of presence data: a performance assessment of methods for correcting sampling bias. Plos One. 9(5):1-13. https://doi.org/10.1371/ journal.pone.0097122 PMID. 24818607. 
Fraitag, G. and Debouck, D. G. 2002. Taxonomy, distribution, and ecology of the genus Phaseolus (Leguminosae-Papilionoideae) in North America, México and Central America. First Edition Botanical Research Institute of Texas. Fort Worth, Texas. USA. 300 p.

Gepts, P. and Debouck, D. G. 1991. Origin, domestication and evolution of the common bean (Phaseolus vulgaris L.). In: common beans. Research for crop improvement. A. van Schoonhoven, O. and Voysest, O. (Eds.). CAB International. Wallingford, United Kingdom. 7-53 pp.

Hijmans, R.; Cameron, S.; Parra, J.; Jones, P. and Jarvis, A. 2005. Very high resolution interpolated climate surfaces of global land areas. Inter. J. Climatol. 25(15):1965-1978.

Instituto de Ecología. 2009. México cuarta comunicación nacional ante la convención marco de las Naciones Unidas sobre cambio climático. Secretaría de Medio Ambiente y Recursos Naturales (SEMARNAT). México, DF. 274 p.

Jarvis, A.; Guevara, E.; Reuter, H. I. and Nelson, A. D. 2008. Hole-filled SRTM for the globe: version 4: data grid. Web publication site. SRTM 90 m DEM digital elevation database. CGIAR Consortium for Spatial Information [http://srtm.csi.cgiar.org/].

Kirchhoff, P. 1943. Mesoamérica: sus límites geográficos, composición étnica y caracteres culturales. Acta Americana. 1(1):92-107.

López, A. J. J.; Lépiz, I. R.; González, E. D. R.; Rodríguez, M. R. y López, A.E. 2016. Variabilidad morfológica de Phaseolus lunatus L. silvestre de la región Occidente de México. Rev. Fitotec. Mex. 39(1):49-58.

López, S. J. L.; Ruiz, C. J. A.; Sánchez, G. J. J. and Lépiz, I. R. 2005. Climatic adaptation of 25 wild bean species (Phaseolus spp.) in Mexico. Rev. Fitotec. Mex. 28(3):221-230.

Martínez-Castillo, J.; Camacho-Pérez, L.; Villanueva-Viramontes, S.; Andueza-Noh, R. H. and Chacon-Sánchez, M. I. 2014. Genetic structure within the Mesoamerican Gene Pool of wild Phaseolus lunatus (Fabaceae) from Mexico as revealed by microsatellite markers: implications for conservation and the domestication of the species. Am. J. Bot. 101(5):851-864.

Maxted, N.; Magos, B. J. and Kell, S. 2013. Resource book for preparation of national conservation plans for crop wild relatives and landraces. University of Birmingham, United Kingdom. $463 \mathrm{p}$.

Paredes, M.; Becerra, V.; Condón, F.; Germán, S. y Barbieri, R. L. 2010. Utilización de los recursos fitogenéticos. En: Estrategia en los recursos fitogenéticos para los países del Cono Sur. IICA. Montevideo: Procisur. 97-116 pp.

Phillips, S. J.; Anderson, R. P. and Schapire, R. E. 2006. Maximum entropy modeling of species geographic distributions. Ecol. Modelling. 190(3-4):231-259.

Rohlf, F. J. 2009. NTSYSpc: numerical taxonomy system. Ver. 2.21c. Exeter Software: Setauket: New York. 43 p.

Ruiz, C. J. A.; Medina, G. G. y García, R. G. E. 2018. Sistema de información agroclimático para México-Centroamérica. Rev. Mex. Cienc. Agríc. 9(1):1-10.

Sánchez, G. J. J.; Ruiz, C. J. A.; Medina, G. G.; Ramírez, O. G.; De la Cruz, L. L.; Holland, B. J.; Miranda, M. R. and García, R. G. E. 2018. Ecogeography of teosinte. PLoS ONE. 13(2):e0192676. https://doi.org/10.1371/journal. pone.0192676.

Serrano-Serrano, M. L.; Andueza-Noh, R. H.; Martínez-Castillo, J.; Debouck, D. G. and Chacón, M. I. 2012. Evolution and domestication of lima bean in Mexico: Evidence from ribosomal DNA. Crop Sci. 52(4):1698-1712. 
Teixeira, E. I.; Fischer, G.; Van Velthuizen, H.; Walter, C. and Ewert, F. 2013. Global hotspots of heat stress on agricultural crops due to climate change. Agric. Forest Meteorol. 170:206-215.

Thuiller, W.; Lafourcade, B.; Engler, R. and Araújo, M.B. 2009. BIOMOD - a platform for ensemble forecasting of species distributions. Ecography. 32(3):369-373.

Trabucco, A. and Zomer, R. J. 2010. Global soil water balance geospatial database. CGIAR Consortium for Spatial Information. CGIAR-CSI GeoPortal. http://www.cgiar-csi.org. 